\title{
Pulse Equivalent Area Based Orientation of a Class of Under- Actuated System under Constraints
}

\author{
ATIF ALI*, AND MOHAMMAD BILAL MALIK* \\ RECEIVED ON 20.01.2016 ACCEPTED ON 11.05.2016 \\ ABSTRACT
}

\begin{abstract}
APEA (Pulse Equivalent Area) approach for orientation control of an under actuated pointing device is proposed. The presented work is a sequel to the earlier work done by the authors for the orientation control of the drill machine. The desired orientation is realized through control pulses during specific roll instants. These pulses are generated through single pair of electromagnets mounted on the housing of the pointing device. Due to practical limitation of the system, there is an additional constraint on the actuating signal. The amplitude of the actuating signal is fixed and the Pulse width or duration of the pulse can vary. A discrete equivalent model of pointing device is also developed. Anovel approach based on PEA is developed since the exact solution of discrete equivalent model under constraints was not possible. The simulations are included to compare proposed technique with the existing technique developed for similar systems. The performance is also shown under both nominal and parameter variations through Monte Carlo simulations.
\end{abstract}

Key Words: Under Actuated Control, Pulse Equivalent Area, Monte Carlo Simulations, Pulse Width Modulation.

\section{INTRODUCTION}

$\mathrm{T}$ he recent development of new type of sensors and actuators on relatively smaller scale opens dimension for further research and experimentation. These sensors and actuators find many applications but requires comprehensive modeling to avoid compromises in form of input constraints and underactuation.

The orientation control of fully actuated rotor, actuated through excitation of stator windings is a well-established technique [1-5]. However, in case of under-actuated systems it becomes challenging and therefore it is one of the favorite topics in field of controls and dynamics [612]. A discrete-time equivalent model was developed for application discussed in [1], by considering one complete revolution of drill bit as a discrete step. The magnitude and phase of actuation pulse is controlled by discrete time controller to achieve desired orientation of the drill bit on the basis of sampled state feedback. The solution provided by [1] is effective in controlling the orientation, but has a major practical limitation. The pulse width of control input is fixed while its magnitude is proportional

*Department of Electrical Engineering, National University of Sciences \& Technology, Islamabad.

Mehran University Research Journal of Engineering \& Technology, Volume 36, No. 3, July, 2017 [p-ISSN: 0254-7821, e-ISSN: 2413-7219] 
to the amount of control force required and is unlimited. However, actuation signal with unlimited amplitudeleads to saturation and stability issues.This can be overcome by assuming the actuation pulse to be of fixed amplitude and variable duration. But this becomes a constraint on an already under actuated system. The discrete time equivalent model of the system is non-closed form thus making the problem more difficult. The controller design for such systems is a real challenge, as the classical control theory is not applicable here.

PEA is applicable to system under discussion [13-15]. It implies that two input signals with different waveforms can result similar outputs if they have the same areas [16]. The concept of PEA is used here to adjust the pulse width and delay of control input. The main advantage of PEA is that it is almost implementable. It is shown via simulations that the presented scheme provided a smooth control for precise movement and overcome the limitations on the control effort. The robustness of the scheme is verified through Monte Carlo simulations.

The remainder of the paper is organized as follows: Section 2 describes the model of the under actuated pointing device. Section 3 presents the discrete time equivalent model. Section 4 covers PEA based scheme followed by the conclusion and references.

\section{SYSTEM DESCRIPTION}

The plant is a laser pointing device and its construction is shown in Fig. 1. The pointing device is mounted on a shaft which spins or rotates about its z-axis, while right and left and up and down movements areabout $\mathrm{x}$-axis and $\mathrm{y}$-axis respectively. A permanent magnet having a single pair of pole mounted on the shaft is responsible for the change of orientation of the device. Two sets of winding on the housing acts as electromagnetic poles. The magnetic field produced by these electromagnetic poles interacts with that of permanent magnet mounted on the spinning shaft produces a torque which in turns is used for changing orientation of the pointing device.Sinceshaft is rotating, therefore, both axes can be excited. But due to single pair of poles only one axis can be excited at one time, thus the fully actuated system convertsinto an under actuated system. The electromagnetic poles are excited for a specific duration at specific roll phase to orient the device in the desired direction.

\section{$2.1 \quad$ Theorem}

The time derivative of a vector $\vec{v}$ with respect to the inertial frame is related to the time derivative with respect to a rotating coordinate frame by [17]:

$\frac{d}{d t_{I}} \vec{v}=\frac{d}{d t_{r o t}} \vec{v}+\vec{\omega}_{r o t} \times \vec{v}$

Where $\vec{\omega}_{\text {rot }}$ is the angular velocity of the rotating coordinate frame and subscript 'I' indicates that the derivative must be obtained with respect to inertial frame with its time derivative with respect to body axes.

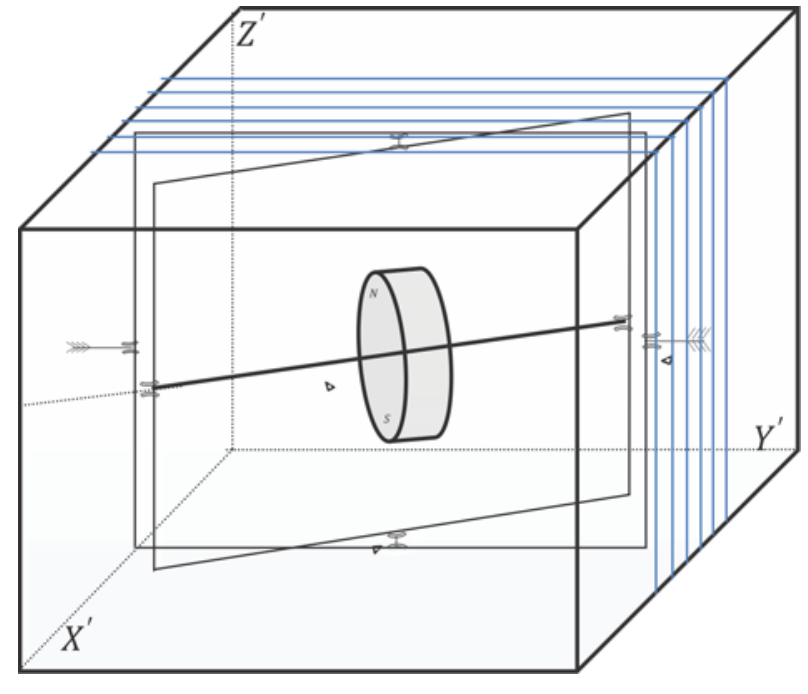

FIG. 1. UNDER-ACTUATED POINTING DEVICE

Mehran University Research Journal of Engineering \& Technology, Volume 36, No. 3, July, 2017 [p-ISSN: 0254-7821, e-ISSN: 2413-7219] 


\subsection{Rotational Dynamics}

The frame of reference attached with the rotor is (X,Y,Z) and the other attached with the stator is ( $\left.\mathrm{X}^{\prime}, \mathrm{Y}^{\prime}, \mathrm{Z}^{\prime}\right)$ as shown in Fig. 2. The magnetic field produced by rotor magnet is always along rotor's Y-axis. There fore the torque produced will always be along rotor's $\mathrm{X}$-axis. As the rotor is spinning, therefore, when $\omega_{\mathrm{z}} \mathrm{t}=0^{\circ}$, the direction of torque is along stator's $\mathrm{X}$ axis and when $\omega_{\mathrm{z}} \mathrm{t}=90^{\circ}$, the direction of torque is alongstator's $Y$ ' axis and so on. According to Newton's second law the net moment acting on a body is equal to the time rate of change of angular momentum $\vec{H}$ and mathematically as:

$\vec{\tau}=\frac{d}{d t_{I}} \vec{H}$

where

$$
\vec{H}=J_{x} \omega_{x} \hat{t}+J_{y} \omega_{y} \hat{J}+J_{z} \omega_{z} \hat{k}
$$

Subscript "I" in Equation (2) indicates that derivative should be obtained with respect to inertial frame. As the stator axis is non-inertial frame therefore, Equation (2) after employing Equation (1) becomes:

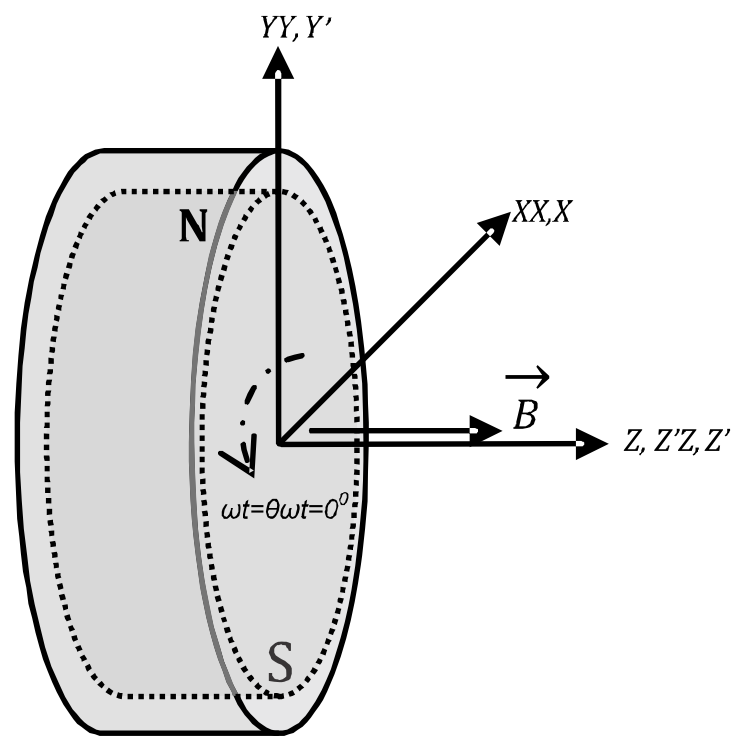

FIG. 2. DIRECTION OF ACTUATION

$$
\vec{\tau}=\frac{d}{d t_{r}} \vec{H}+\left(\vec{\omega}_{r} \times \vec{H}\right)
$$

Subscript "r" in Equation (4) indicates rotor frame. The Euler equations in the rotor frame of reference are given as:

$$
\begin{aligned}
& \tau_{x}=J_{x} \frac{d \omega_{x}}{d t}-\left(J_{y}-J_{z}\right) \omega_{y} \omega_{z} \\
& \tau_{x}=J_{y} \frac{d \omega_{y}}{d t}-\left(J_{z}-J_{x}\right) \omega_{z} \omega_{x} \\
& \tau_{z}=J_{z} \frac{d \omega_{z}}{d t}-\left(J_{x}-J_{y}\right) \omega_{x} \omega_{y} \\
& \tau=\tau_{x} \hat{\imath}+\tau_{y} \hat{J}+\tau_{z} \hat{k}
\end{aligned}
$$

The torques and angular velocities in the rotor frame need to be transformed into stator frame. Using Fig. 3, the relation between the torques in two frames is given as:

$$
\begin{aligned}
& {\left[\begin{array}{l}
\tau_{x} \\
\tau_{y}
\end{array}\right]=\left[\begin{array}{cc}
\cos \theta & \sin \theta \\
-\sin \theta & \cos \theta
\end{array}\right]\left[\begin{array}{c}
\tau_{x}^{\prime} \\
\tau_{y}^{\prime}
\end{array}\right]} \\
& {\left[\begin{array}{l}
\omega_{x} \\
\omega_{y}
\end{array}\right]=\left[\begin{array}{cc}
\cos \theta & \sin \theta \\
-\sin \theta & \cos \theta
\end{array}\right]\left[\begin{array}{c}
\omega_{x}^{\prime} \\
\omega_{y}^{\prime}
\end{array}\right]}
\end{aligned}
$$

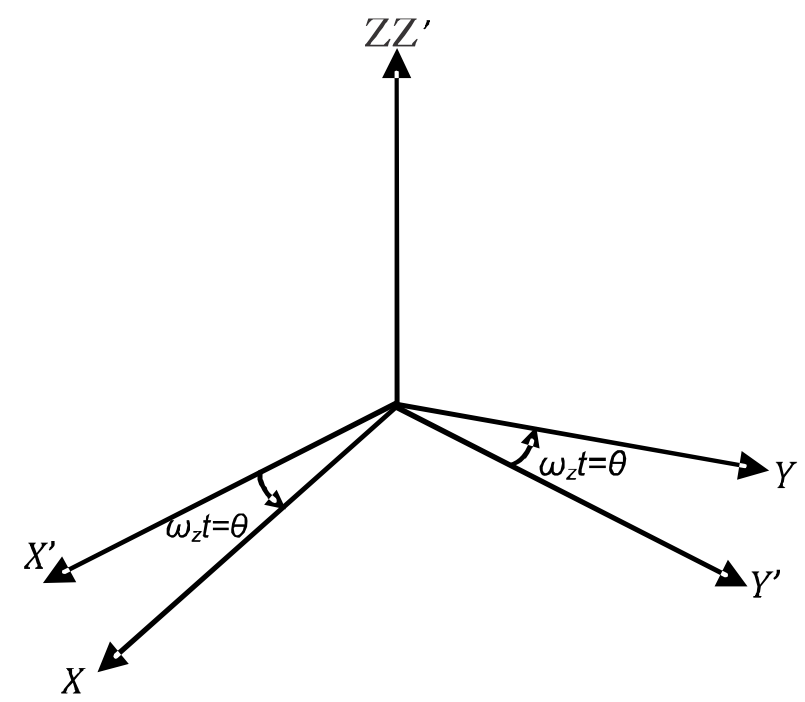

FIG. 3. FRAME TRANSFORMATION [18]

Mehran University Research Journal of Engineering \& Technology, Volume 36, No. 3, July, 2017 [p-ISSN: 0254-7821, e-ISSN: 2413-7219] 
Letting $\mathrm{J}_{\mathrm{x}}=\mathrm{J}_{\mathrm{y}}=\mathrm{J}$ due to axis symmetry, after some algebraic manipulations in Equations (5-6), we get torques fixed with housing as:

$\tau_{x}^{\prime}=J_{x} \dot{\omega}_{x}^{\prime}+J_{z} \omega_{z} \dot{\omega}_{y}^{\prime}$

$\tau_{y}^{\prime}=J_{x} \dot{\omega}_{y}^{\prime}+J_{z} \omega_{z} \omega_{x}^{\prime}$

$\tau_{z}=\tau_{z}=J_{z} \dot{\omega}_{z}$

No torque is applied to the spin axis as it coincides with Z axis therefore $\tau_{\mathrm{z}}^{\prime}=0$. The angular momentum along $\mathrm{Z}$ axis is $\mathrm{H}_{\mathrm{z}}=\mathrm{C} \omega_{\mathrm{z}}$. The presence of damping forces due to friction etc. will cause a damping torque about $X^{\prime}$ and $Y^{\prime}$ axis [19].

Therefore, Equation (7) reduces to Equation (8):

$k_{i} \tau_{x}^{\prime}=J_{x} \dot{\omega}_{x}^{\prime}+b \omega_{x}^{\prime}+H_{z} \omega_{y}^{\prime}$

$k_{i} \tau_{y}^{\prime}=J_{x} \dot{\omega}_{y}^{\prime}+b \omega_{y}^{\prime}+H_{z} \omega_{z}^{\prime}$

Due to single pole mounted on the rotor, the torque will be availableonly in one channel i.e. $\tau_{\mathrm{x}}=\mathrm{t}$ and $\tau_{\mathrm{y}}=0$ and Equation (6) reduces as:

$\tau_{x}^{\prime}=\tau \cos \omega t$

$\tau_{\mathrm{y}}=\tau \sin \omega \mathrm{t}$

After employing Equation (9) in Equation (8), we have:

$J_{z} \ddot{\theta}_{x}^{\prime}+b \ddot{\theta}_{x}^{\prime}+H_{z} \ddot{\theta}_{y}^{\prime}=k_{i} \tau \cos \omega t$

$J_{x} \ddot{\theta}_{y}^{\prime}+b \ddot{\theta}_{y}^{\prime}+H_{z} \ddot{\theta}_{x}^{\prime}=k_{i} \tau \sin \omega t$

To represent Equation (10) in state space form, $\theta_{\mathrm{x}}^{\prime}$ and $\theta_{\mathrm{y}}^{\prime}$ are represented as $\mathrm{x}_{3}$ and $\mathrm{x}_{4}$ and $\theta_{\mathrm{x}}^{\prime}$ and $\theta_{\mathrm{y}}^{\prime}$ as $\mathrm{x}_{3}$ and $\mathrm{x}_{4}$. The applied torque is represented as u. Respective angular positions are obtained by integrating angular velocities, which can be accuratelycalculated usingEuler angle transformations [19]. Finally the state space representation in housing frame of reference is given in Equation (11).

$$
\begin{aligned}
& \dot{x}_{1}=-\frac{b}{J_{x}} x_{1}-\frac{H_{2}}{J_{x}} x_{2}+\frac{k_{i}}{J_{x}} u_{1} \\
& \dot{x}_{2}=\frac{H_{z}}{J_{x}} x_{1}-\frac{b}{J_{x}} x_{2}+\frac{k_{i}}{J_{x}} u_{2} \\
& \dot{x}_{3}=x_{1} \\
& \dot{x}_{4}=x_{2}
\end{aligned}
$$

The Equation (11) can be written in matrix form Equation (12), the output vector $y(t)$ is formed by $x_{3}$ and $x_{4}$ states and the input $\mathrm{u}(\mathrm{t})$ is given by Equation (13). The output vector $y(t)$ comprises of angular positions $\theta_{x}$ and $\theta_{y}$ in stator frame of reference.

$$
\begin{aligned}
& \dot{x}(t)=A x(t)+B u(t) \\
& y(t)=C x(t) \\
& u(t)=\left[\begin{array}{c}
\tau \cos \omega t \\
\tau \sin \omega t
\end{array}\right] C x(t)
\end{aligned}
$$

where

$$
\bar{A}=\left[\begin{array}{cccc}
-\frac{b}{J_{x}} & -\frac{H_{z}}{J_{X}} & 0 & 0 \\
-\frac{H_{Z}}{J_{x}} & -\frac{b}{J_{x}} & 0 & 0 \\
1 & 0 & 0 & 0 \\
0 & 1 & 0 & 0
\end{array}\right], \bar{B}=\left[\begin{array}{c}
\frac{K_{i}}{J_{x}} \cos \omega t \\
\frac{K_{i}}{J_{x}} \sin \omega t \\
0 \\
0
\end{array}\right], \bar{C}=\left[\begin{array}{llll}
0 & 0 & 1 & 0 \\
0 & 0 & 0 & 1
\end{array}\right]
$$

\subsection{Actuation Constraints}

The actuation in control scheme [1] is rectangular pulses of fixed duration $\mathrm{L}$ and variable amplitude as shown in Fig. 4. The center of the actuation pulse is the point where the magnetic field of the rotor and the precession axis are precisely aligned. In each actuation cycle, the input pulse is applied after a time delay $\beta$ and the system remains unactuated for the rest of cycle i.e. T-L. The requirement of pulse width having unlimited amplitude is restricted to maximum amplitude. This limitation was overcome by assuming the actuation pulse having fixed amplitude with variable duration. The modified actuation cycle of period Tapplied for a complete revolution is shown in Fig. 5. The

Mehran University Research Journal of Engineering \& Technology, Volume 36, No. 3, July, 2017 [p-ISSN: 0254-7821, e-ISSN: 2413-7219] 
phase of actuation $\Delta$ represents the shift of the center of the rectangular pulse from the start of the revolution. The amplitude $\alpha$ of the pulse is fixed and PWvaries according to the requirement of the control signal magnitude. This marks the difference in approach from [1] where a pulse having fixed width with varying amplitude was used for the actuation. Therefore, $\mathrm{P}$ Wand $\Delta$ are to be estimated subject to the following constraints:

$$
\begin{aligned}
& t_{1} \leq|P W| \leq t_{2} \\
& 0 \leq \Delta \leq T_{s} \\
& \|u(t)\|=\alpha
\end{aligned}
$$

Where

$$
\left(t_{2}-t_{1}\right) \leq \frac{T_{s}}{2}
$$

\section{DISCRETE TIME EQUIVALENT MODEL}

The discrete-time equivalent model of the given plant is derived using Fig. 4. The time interval $[\mathrm{KT},(\mathrm{K}+1) \mathrm{T}]$ is considered as one revolution in the rotor frame. The equivalent discrete model is developed in following two steps:

Interval-l I

$\mathrm{t} \in[\mathrm{KT}+\beta, \mathrm{KT}+\mathrm{L}+\beta]$

In the first interval, the system Equation (12) is actuated $x(K T+L+\beta)=A_{d 1} x(K T)+B_{d 1} u(K T+\beta)$

Where

$$
A_{d 1}=e^{A(L)}, B_{d 1}=B \int_{K T+\beta}^{K T+\beta+L} e^{A(K T+\beta+L-\phi)} d \phi
$$

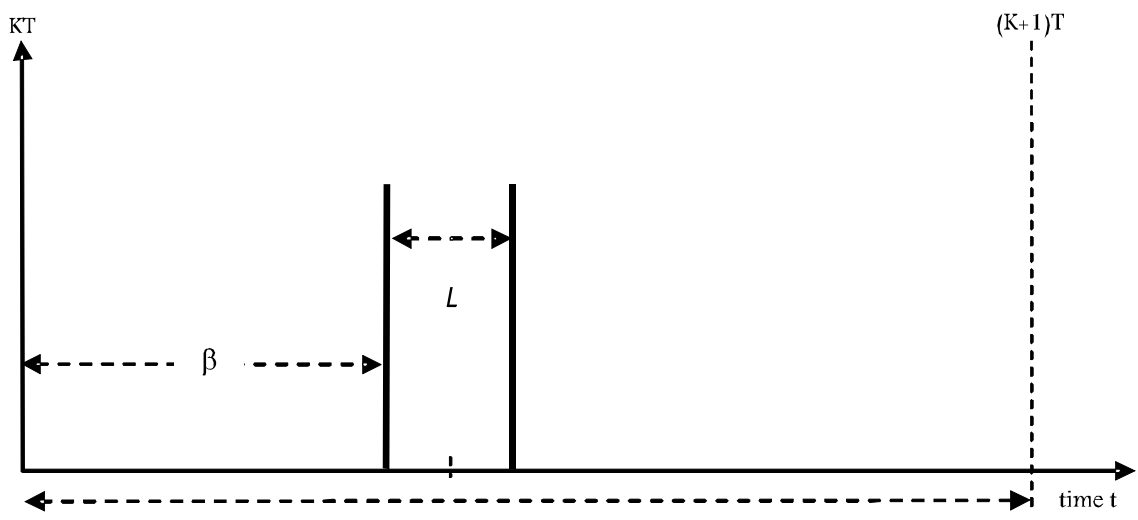

FIG. 4. SINGLE ACTUATION CYCLE AMPLITUDE MODULATION [1]

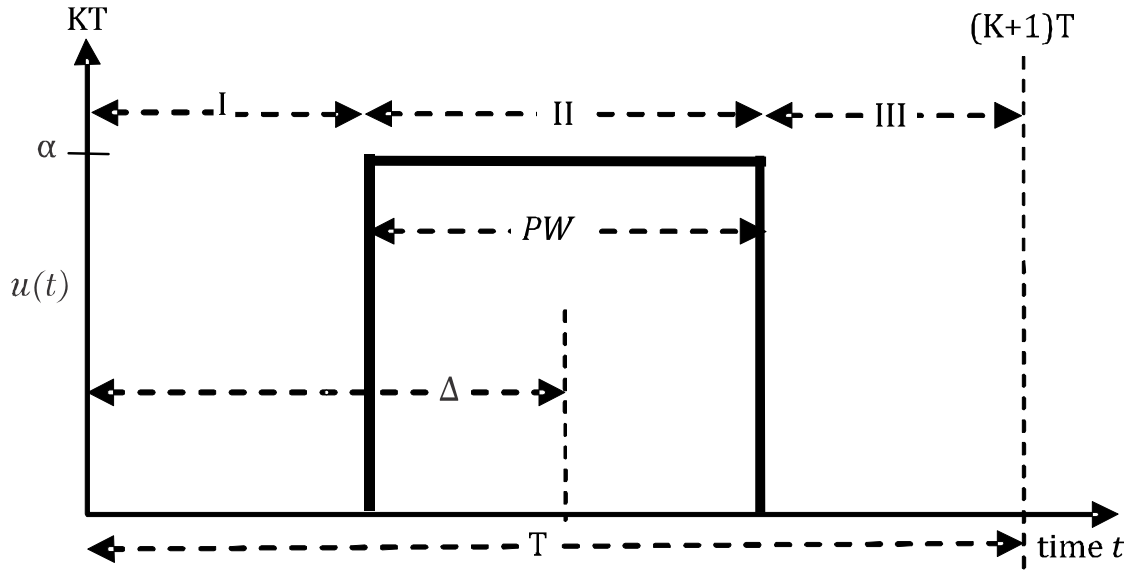

FIG. 5. SINGLE ACTUATION CYCLE OF PERIOD T UNDER CONSTRAINTS 
Interval -II

$\mathrm{t} \in[\mathrm{KT}+\beta+\mathrm{L}, \mathrm{KT}+\mathrm{L}+\beta]$

The system remains unactuated in this interval and the states are given by:

$x(K T+T+\beta)=e^{A(T-L)} x(K T+\beta+L)$

by substituting value of $x(\mathrm{KT}+\mathrm{L}+\beta]$ in above equation we have:

$x(K T+T+\beta)=A_{d} x(K T+\beta)+B_{d} u(K T+\beta)$

Where

$A_{d}=e^{A(T-L)} A^{d 1}, B_{d}=e^{A(T-L)} B_{d 1}$

\subsection{Discrete Time Equivalent Model}

The discrete-time equivalent model of the given plant for actuation signal under constraints Equation (15) is derived using Fig. 5. The time interval [KT,(K+1)T] is considered as one revolution in the rotor frame. The time period $\mathrm{T}$ is divided into three separate intervals i.e. intervalI, II and III. It must be noted that during interval-I and III the system is un-actuated.

Interval-1 I

$t \in\left[K T, K T+\Delta-\frac{P W}{2}\right]$

The system Equation (12) is unactuated and the states are given as:

$x\left(K T+\Delta-\frac{P W}{2}\right) e^{A\left(K T+\Delta-\frac{P W}{2}\right)} x(K T)$

Interval-II

$t \in\left[K T+\Delta-\frac{P W}{2}, K T+\Delta+\frac{P W}{2}\right]$
The system Equation (12) is actuated and the states are given as:

$x\left(K T+\Delta+\frac{P W}{2}\right)=e^{A P W} \mathrm{e}^{\mathrm{A}\left(\Delta-\frac{\mathrm{PW}}{2}\right)} \mathrm{x}(\mathrm{KT})+\int_{-P W / 2}^{P W / 2} e^{A\left(\frac{P W}{2}-\phi\right)} d \phi \mathrm{Bu}$

Interval-III

$t \in\left[K T+\Delta-\frac{P W}{2},(K T+1)\right]$

The system Equation (12) is unactuated during this interval and the states are given as:

$x((K+1) T)=e^{A\left(T-\Delta-\frac{P W}{2}\right)} x\left(K T+\Delta+\frac{P W}{2}\right)$

Substituting values from Equation (17) into Equation (18) we get:

$$
x((K T+1) T)=e^{A T} x(K T)+e^{A(T-\Delta)} \int_{-P W / 2}^{P W / 2} e^{-A \varphi} d \varphi \varphi B
$$

Due to presence of integral in Equation (19) its generalized closed form Equation (20) is not possible. Byusing Taylor series an approximate non-closed form expression is obtained. The approximate states at time $(\mathrm{T}+1)$ for a specific PW and $\Delta$ are given in Equation (21) as follows:

$$
x((K+1) T) \cong e^{A T} x(K T)+e^{A(T-\Delta)}\left(P W+\frac{A^{2} P W^{3}}{24}+\frac{A^{4} P W^{5}}{1920}\right) B u \text { (20) }
$$

\section{PEA BASD CONTROL SCHEME}

As evident from Equation (19), that the exact solution through discrete time equivalent model cannot be obtained under constraints Equation (15). Therefore, PEA based control scheme is applicable to the system constraints and under discussion is shown in Fig. 6. 
The exact solution Equation (16) is transformed into PEA signal through Equation (21) thus complying constraints Equation (15).

$$
P W=\frac{L U}{\alpha}, \Delta=\beta+\frac{L}{2}
$$

Where PEA is based on the principle for two input signals of different waveforms, similar outputs can be generated if they have the same areas [16]. When this concept is applied to a PWM (Pulse Width Modulation) signal whose pulse width is to be modified to achieve a PEA equivalent of a given control signal then the relationship is governed by [16]:

$$
\int_{K T}^{(K=1) T} w(t) d t=U \sigma_{k}
$$

$\mathrm{U}$ is pulse amplitude, $\mathrm{T}$ is the PEA interval, $\mathrm{u}(\mathrm{t})$ is given control signal to be converted into pulse signal and $\alpha_{k}$ is pulse-width to be determined.

\subsection{Controller Design}

The overall control scheme is shown in Fig. 7. Controller is based on the discrete equivalent model given by Equation (16). The standard pole placement technique is utilized for this purpose [20]. The control input is:

$\mathrm{U}[\mathrm{k}]=-\mathrm{Fx}[\mathrm{k}]+\mathrm{Nr}$

The matrix $F$ is chosen such that the eigenvalues of $\left(A_{d}-\right.$ $\left.B_{d} F\right)$ are within the unit circle. The reference signal is $\left(r_{x}, y_{y}\right) \in R_{2}$, where matrix $N$ is $\left.C\left[I-A_{d}+B_{d} F\right)^{-1} B_{d}\right]^{-1}$

\subsection{Simulations and Performance}

Simulations were carried out in MATAB and SIMULINK. The parameters used are $\omega=400 \pi \mathrm{rad} / \mathrm{sec}, \mathrm{b}=400, \mathrm{k}_{\mathrm{i}}=1$, $\mathrm{J}=4 \mathrm{~kg}, \mathrm{~J}_{\mathrm{z}}=5 \mathrm{~kg}, \mathrm{~F}=[0.992,0.995+9 \mathrm{j}, 0.99,0.99+9 \mathrm{j}]$. The time interval to complete one revolution is taken to be 0.05 sec. A comparison of old technique [1] and PEA base technique has been carried out. Fig. 8(a) and Fig. 9(a)
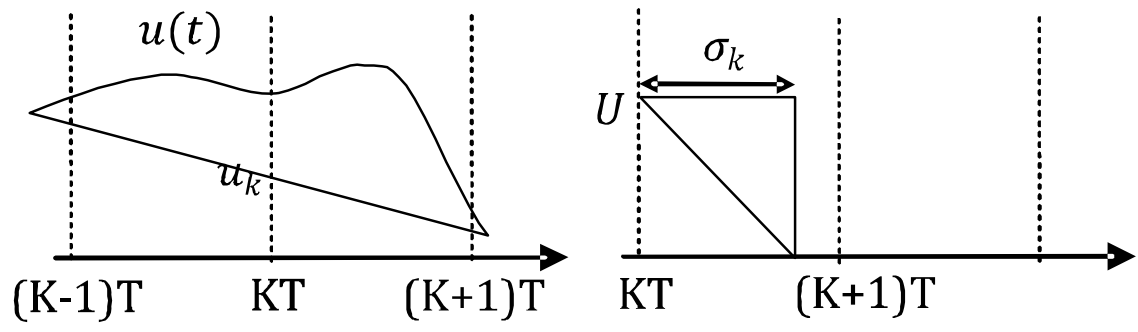

FIG. 6. PEA BASED PULSE WIDTH DETERMINATION [16]

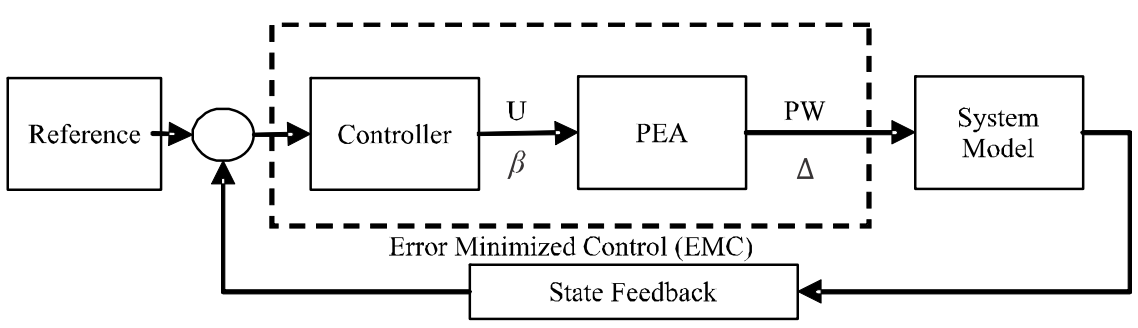

FIG. 7. PEA BASED CONTROL SCHEME

Mehran University Research Journal of Engineering \& Technology, Volume 36, No. 3, July, 2017 [p-ISSN: 0254-7821, e-ISSN: 2413-7219] 
shows the performance of both techniques when the reference to be tracked is constant. It can be seen that both techniques show satisfactory results, however, technique [1] shows better transient performance. Both the techniques were also subjected to time varying reference signals as shown in Fig. 8(b) and Fig. 9(b), but the technique [1] could not follow the desired trajectory whereas PEA based technique follow the varying reference.
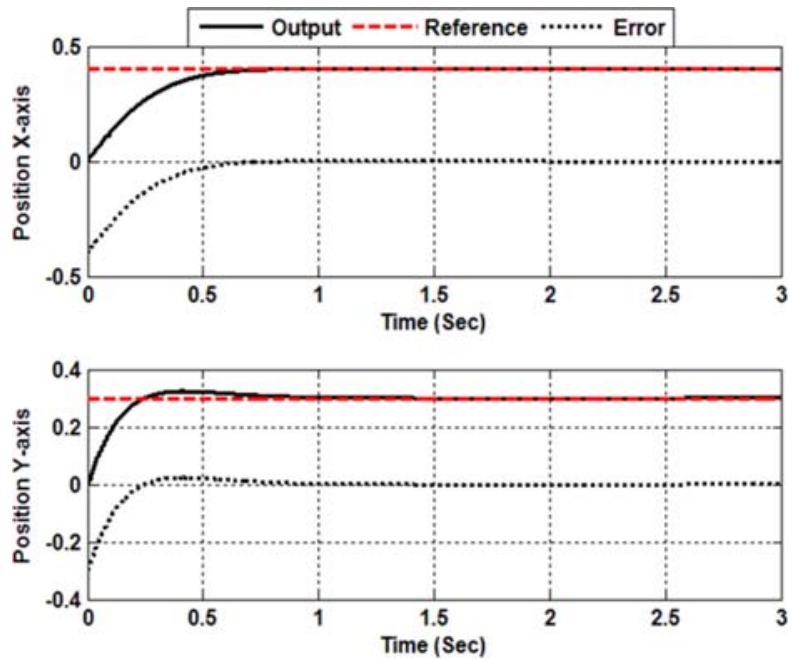

(a) FIXED REFERENCE
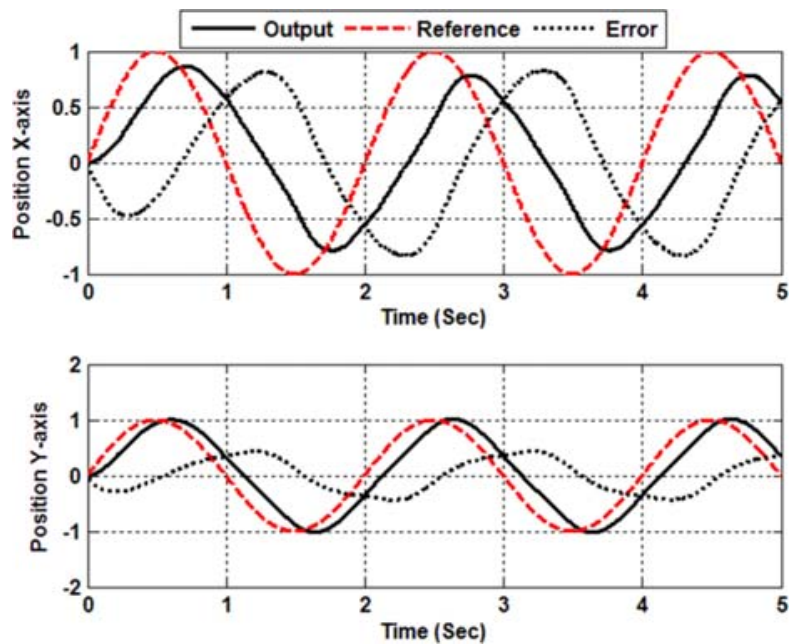

(b) TIME VARYING REFERENCE

FIG. 8. STABILIZATION BASED ON TECHNIQUE [1]

\subsection{Parametric Variation Simulations}

The parametric variation is performed to verify the robustness of proposed technique. A variation of 10 and $25 \%$ was made in the nominal values of the plant.This variation of 10 and $25 \%$ were made in all the parameters of Equation (14) simultaneously. As seen from the results in Fig. 10 that the system remains stable with minor variations in settling time. The transient performance deteriorates at $25 \%$ parametric variations but still remains
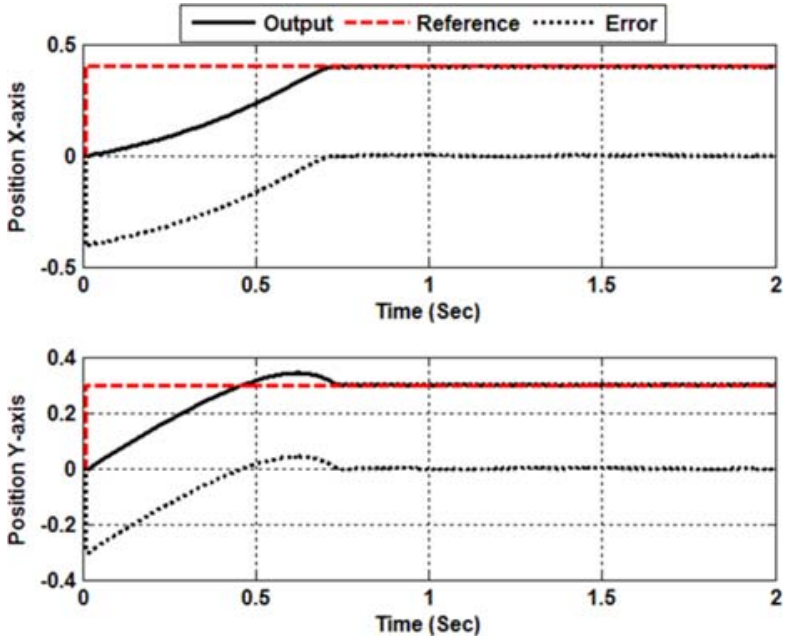

(a) FIXED REFERENCE
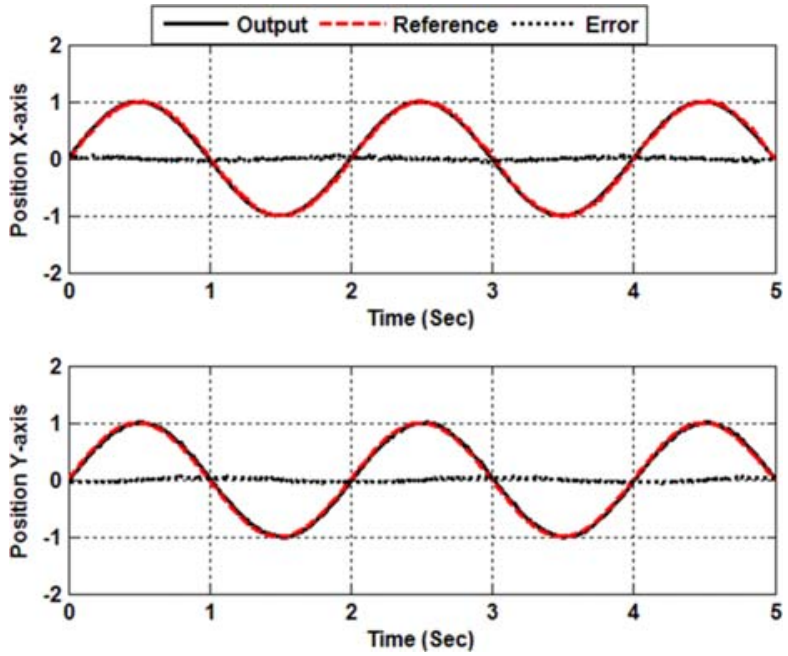

(b)TIME VARYING REFERENCE

FIG. 9. PEA BASED STABILIZATION 

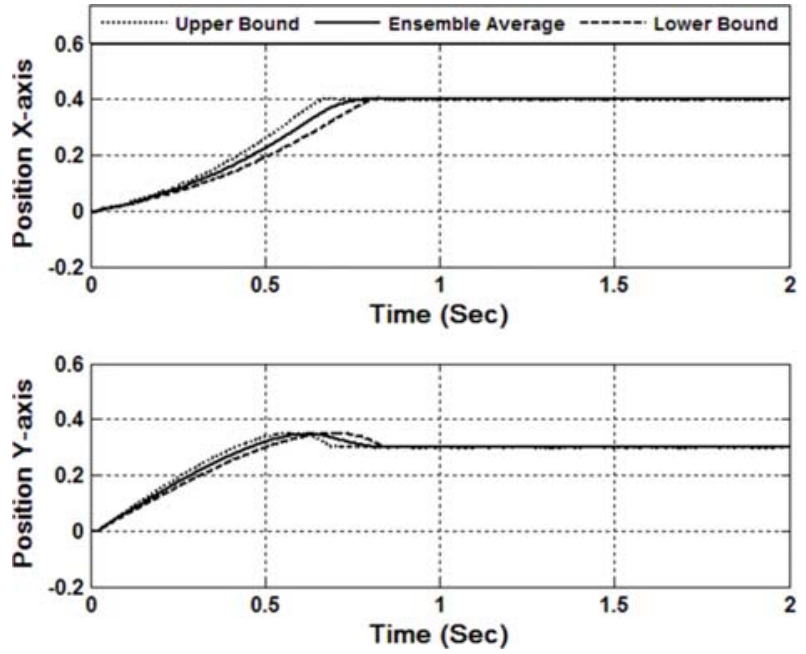

(a) UNDER 10\%PARAMETRIC VARIATION
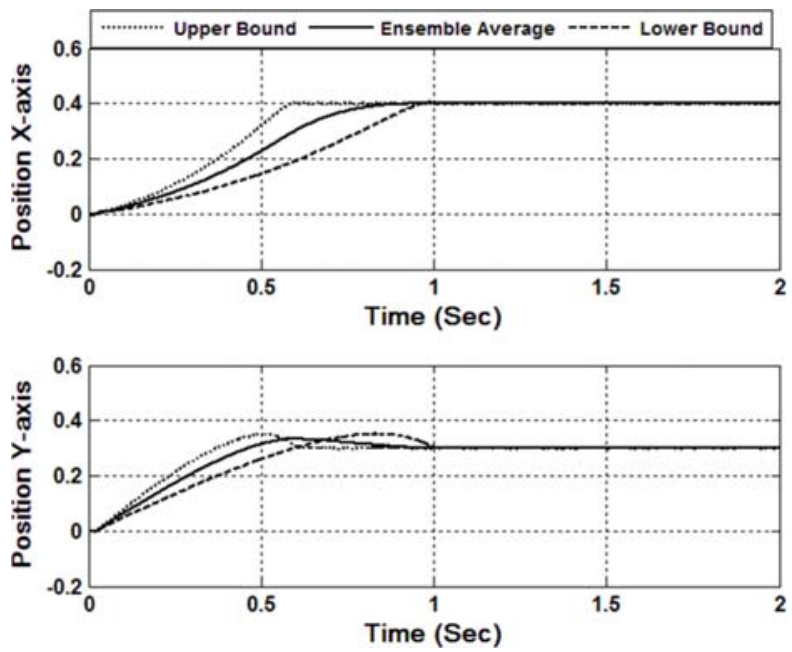

(b) UNDER 25\% PARAMETRIC VARIATION

FIG. 10. PEA BASED STABILIZATION

stable. To sum up the overall system performance with PEA based technique is robust enough to handle perturbations and disturbances in plant parameters.

\section{CONCLUSIONS}

A novel PEA based scheme is presented for orientation control of an under-actuated pointing device under input constraints. A single pair of electromagnet is used to control two axes movement due to which the system becomes under actuated. The solution has a major practical limitation as the pulse width of control input is fixed while its magnitude is proportional to the amount of control force required and is unlimited. To overcome this limitation two constraints are applied on actuating signal of fixed amplitude with adjustable pulse width. Due to the given constraints the exact solution of discrete time equivalent model was not possible. Therefore, control signal generated is transformed into a PEA signal which ensures that the control signalis so produce to meet the constraints. The proposed technique has shown satisfactory results inachieving the desired orientation for the nominal as well as for the perturbed system with 10 and $25 \%$ variation in parameters.

The main advantage of PEA is that it is almost implementable. Although PEA concept is almost implementable but is based on approximation, therefore to achieve precise control with this technique is difficult to achieve. Another limitation is of high modulation frequency as the averaging response becomes closer to the given control signal at high frequencies. The higher model formulas are more difficult to convert using PEA. The minimum pulse width which cannot be ignored is a major limitation for developing exact PEA signal.

\section{FUTURE WORK}

The future work may be focused on the novel optimization based technique so that precise control can be achieved in more efficient way.

\section{NOTATIONS}

$\mathrm{X}, \mathrm{Y}, \mathrm{Z} \quad$ System of rectangular body fixed axes

$X^{\prime}, Y^{\prime}, X^{\prime} \quad$ System of rectangular axes fixed to housing

$\mathrm{H}_{\mathrm{z}} \quad$ Angular momentum along $\mathrm{Z}$ axis

$\tau_{x}, \tau_{y}, \tau_{z} \quad$ Torque vector along $\mathrm{X}, \mathrm{Y}, \mathrm{Z}$ axis

$\theta_{\mathrm{x}}^{\prime}, \theta_{\mathrm{y}}^{\prime} \quad$ Angular positions about $\mathrm{X}^{\prime}$ and $\mathrm{Y}^{\prime}$ axes

$\omega_{\mathrm{x}}, \omega_{\mathrm{y}}, \omega_{\mathrm{x}}$ Angular spin velocity along $\mathrm{X}, \mathrm{Y}, \mathrm{Z}$ axis in radians per second

$\bar{B} \quad$ Magnetic field due to stator winding

$\mathrm{J}_{\mathrm{x}}, \mathrm{J}_{\mathrm{y}}, \mathrm{J}_{\mathrm{z}} \quad$ Moment of inertia along X,Y,X axis

b Coefficient of friction

Torque constant

Mehran University Research Journal of Engineering \& Technology, Volume 36, No. 3, July, 2017 [p-ISSN: 0254-7821, e-ISSN: 2413-7219] 


\section{ACKNOWLEDGEMENTS}

This work was part of Ph.D. research work of first authors and was fully supported by the College of Electrical \& Mechanical Engineering, National University of Sciences \& Technology, Islamabad, Pakistan.

\section{REFERENCES}

[1] Malik, M.B., Malik, F.M., and Munawar, K., “Orientation Control of a 3D Under-Actuated Drill Machine Based on Discrete-Time Equivalent Model”, International Journal Robotics and Automation, Volume 27, No. 4, [DOI:10.2316/Journal.206.2012.4.206-3324], 2012.

He, J., Gao, F., and Zhang, D., "Design and Performance Analysis of a Novel Parallel Servo Press with Redundant Actuation”, International Journal of Mechanics and Materials in Design, Volume 10, No. 2, pp. 145-163, 2014.

[3] Criens, C., Willems, F., and Steinbuch, M., "Under Actuated Air Path Control of Diesel Engines for Low Emissions and High Efficiency”, Proceedings of World Automotive Congress, Volume 189, pp. 725-737, 2013.

Dong, W., Gu, G.Y., Zhu, X., and Ding, H., "Solving the Boundary Value Problem of an Under-Actuated Quadrotor with Subspace Stabilization Approach”,Journal of Intelligent and Robotic Systems, [DOI: 10.1007/ s10846-014-0161-3], 2014.

Bedereson, R., Wallace, R., and Schwartz, E., “A Miniature Pan-Tilt Actuator; the Spherical Pointing Motor”, IEEE Trnasactons on Robotics and Automation, Volume 10, pp. 298-308, 1994.

[6] Liu, Y., and Yu, H.,"A Survey of Underactuated Mechanical Systems", Control Theory Applied, Volume 7, pp. 921-935, 2013.

Pucci, D., Romano, F., and Nori, F., “Collocated Adaptive Control of Underactuated Mechanical Systems”, IEEE Transactions on Robotics, Volume 31, No. 6 pp. 1527-1536, 2015.

[8] Khan, Q.S., and Akmeliawati, R., "Robust Control of Underactuated Systems: Higher Order Integral Sliding Mode Approach", Mathematical Problems in Engineering, Volume 11, [DOI:10.1155/2016/5641478], 2016 .
Xuan, H.T., Chemori, A., Anh, T.P., Xuan, H.L., Hoai, T.P., and Viet. P.V., "From PID to L1 Adaptive Control for Automatic Balancing of a Spacecraft Three-Axis Simulator”, International Journal of Emerging Technology and Advanced Engineering, Volume 6, No. 1, pp. 77-86, 2016.

[10] Maalouf, D., Chemori, A., and Creuze, V., "L1 Adaptive Depth and Pitch Control of an Underwater Vehicle with Real-Time Experiments”, Ocean Engineering (Elsevier), [DOI: 10.1016/j.oceaneng], pp. 66-77, 2015.

[11] Rifai, H., Abdessalem, M.S.B., Chemori, S., Mohammed, D., and Amirat. Y., "Augmented L1 Adaptive Control of an Actuated Knee Joint Exoskeleton: From Design to Real-Time Experiments”, IEEE Stockholm, Sweden, 2016.

[12] Aissa, C.Z., Laleg,T.M., and Chemori, A., "Control of a Perturbed Under-Actuated Mechanical System”, IEEE Multi Conference on Systems and Control, Sydney, Australia, 2015.

[13] Andeen,R.E., "The Principle of Equivalent Areas", Transactions on AIEE, Volume 79, pp. 332-336, 1960.

[14] Sakamoto, T., and Hori, N., "New PWM Schemes Based on the Principle of Equivalent Areas”, IEEE International Symposium on Industrial Electronics, pp. 505-510, L’Aquila, Italy, 2002.

[15] Barr, M., "Pulse Width Modulation: Embedded Systems Programming”, pp. 103-104, September, 2001.

[16] Suzuki, T., Ueno, T., and Hori, N., “ Experimental Verification of Pea-Based PWM Control using On-Off Type Air-Jet Thrusters”, Proceedings of International Conference on Information and Automation, Colombo, Sri Lanka, December 15-18, 2005.

[17] Stevens, B.L., and Lewis, F.L., "Aircraft Control and Simulation”, 2 2d Edition, Wiley, USA,2003.

[18] Savet, P.H., "Gyroscopes Theory and Design with Applications to Instrumentation, Guidance and Control”, McGraw-Hill, 1961.

[19] Longuski, J.M., "Solution of Euler's Equations of Motion and Eulerian Angles for Near Symmetric Rigid Bodies Subject to Constant Moments”, AIAA/AAS Astrodynamics Conference, Massachusetts, USA, August 11-13, 1980.
Rugh,W.J., "Linear System Theory”, $2^{\text {nd }}$ Edition, Prentice Hall, 1996. 